4th International Conference Photoinduced Phase Transitions and Cooperative Phenomena, Wrocław 2011

\title{
Photo-Induced Structural Changes at a Surface of Organic Single Crystals Observed by Vibrational Sum Frequency Generation Spectroscopy
}

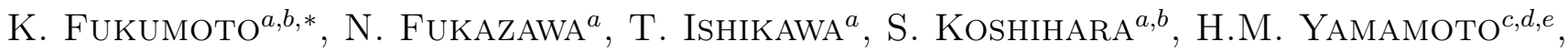 \\ R. KATO ${ }^{c}$ AND K. ONDA ${ }^{f}$ \\ ${ }^{a}$ Department of Materials Science, Tokyo Institute of Technology, Meguro-ku, Tokyo 152-8551, Japan \\ ${ }^{b}$ JST-CREST, Kawaguchi, Saitama 332-0012, Japan \\ ${ }^{c}$ RIKEN, Wako, Saitama 351-0198, Japan \\ ${ }^{d}$ JST-PRESTO, Kawaguchi, Saitama 332-0012, Japan \\ ${ }^{e}$ Department of Elec. Chem., Tokyo Institute of Technology, Nagatsuta, Yokohama, 226-8502, Japan \\ ${ }^{f}$ Department of Environ. Chem. and Engineering, Tokyo Institute of Technology \\ Nagatsuta, Yokohama 226-8502, Japan
}

\begin{abstract}
Phase transitions from insulator to metal induced by thermal excitation and by photo-irradiation at the surface of $\beta^{\prime}$-(BEDT-TTF)(TCNQ) are investigated using vibrational sum frequency generation spectroscopy. The results are compared with linear reflectivity measurements which detect the information mainly from bulk. IR absorption spectra of $a_{\mathrm{g}}$ vibrational modes of TCNQ induced by the dimerization are observed using vibrational sum frequency generation spectroscopy, and its intensity is found to become smaller by thermal excitation indicating the transition to the metal phase. Time-dependent degree of dimerization is also observed by pump-probe experiments with almost the same statistics as the static measurements. On the other hand, in the reflectivity experiments, the reduction of dimerization is also observed by elevating the temperature, however, the spectral shapes are deformed for the time-resolved measurements. These results imply that the different dynamics occur at the surface and in the bulk.
\end{abstract}

PACS: 78.40.Me, 68.35.Rh, 68.35.Ja

\section{Introduction}

Some of the Mott insulating single crystals show the phase transition to metal or to superconducting phases by changing temperature and/or pressure [1], by applying electric field [2], by electric current [3], or by photo-irradiation [4]. To make these molecular crystals work as (photo-switchable) memory devices, the electric conductivity at the surface has to be controlled, like semiconductor-based devices.

We performed linear reflectivity and vibrational sum frequency generation (VSFG) spectroscopy measurements to investigate difference or similarity of the phase transitions occurring inside the bulk and at the surface of a Mott insulating organic single crystal, $\beta^{\prime}$-(BEDT-TTF) (TCNQ). The crystal is composed of two-dimensional conducting sheets of BEDT-TTF and one-dimensional TCNQ columns. The stacking directions of them are perpendicular to each other. It is Mott insulating at room temperature and turns to be metal with the transition temperature of $330 \mathrm{~K}[1,5]$.

To observe the vibrational spectra only from the sur-

\footnotetext{
* corresponding author; e-mail: fukumoto.k.ab@m.titech.ac.jp
}

face, we have specially tuned the VSFG system. Since SFG is based on the second order non-linear optical susceptibility $\left(\chi^{(2)}\right)$, no signal will be obtained from materials with inversion symmetry. Even for such materials, however, the symmetry is broken at the surface and $\chi^{(2)}$ becomes non-zero [6]. To detect such weak signals without damaging the surface structures, the pulse laser with a high repetition rate and moderate intensity was used.

In this contribution we show the vibrational spectra obtained from the surface and the bulk, and also show the difference of the spectra measured below and above the transition temperature. Structural changes by photo-irradiation are also observed by the pump-probe experiments.

\section{Experiments}

The experimental setup of the VSFG measurements are described elsewhere [7]. The laser pulse with the repetition rate of $1 \mathrm{kHz}$ and the center wavelength of $800 \mathrm{~nm}$ is provided by a mode-locked Ti:sapphire laser and a regenerative amplifier. In the amplifying process, the pulse width is stretched to 4 ps which gives a high energy resolution of $\approx 10 \mathrm{~cm}^{-1}$. The laser pulse is split into three. One is converted into frequency-tunable IR pulse ranging 
from 1000 to $3500 \mathrm{~cm}^{-1}$ by an optical parametric amplifier with difference frequency generation. One out of the other two lasers with the wavelength of $800 \mathrm{~nm}$ is used to generate SFG together with the IR laser. The other $800 \mathrm{~nm}$ pulse is used to pump the phase transition.

Three pulses are introduced to the sample (Fig. 1). The incidence angle of the three is about $45^{\circ}$ from the sample surface and about $5^{\circ}$ away from each other. VSFG signal generated between the IR and $800 \mathrm{~nm}$ lasers is detected by a photomultiplier with through filters and a monochromator. $\beta^{\prime}$-(BEDT-TTF)(TCNQ) crystals are oriented as the stacking direction of TCNQ molecules ( $c$ axis) being almost parallel to the direction given by the projection of the $800 \mathrm{~nm}$ laser to the surface plane (Fig. 1). Therefore using the $p$ polarized laser, VSFG is sensitive to detect the $a_{\mathrm{g}}$ modes induced by the dimerization of TCNQ molecules [5]. Time-resolved infrared vibrational spectra in reflectivity are measured using a broadband femtosecond mid-infrared pulse and a MCT $(\mathrm{HgCdTe})$ linear array detector [8], and the polarization of light is aligned to detect the TCNQ dimerization. The crystal is grown by evaporating the solvent slowly at room temperature and is obtained with black in color and in an elongated-shape $[1,9]$.

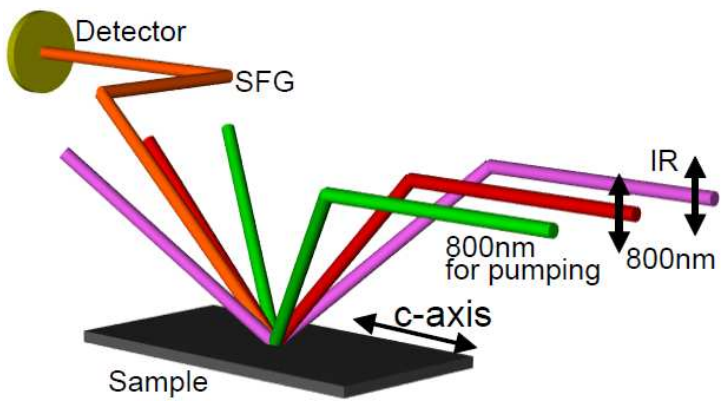

Fig. 1. Experimental geometry of VSFG. IR and one out of two $800 \mathrm{~nm}$ laser pulses are used to generate SFG signal, and the other $800 \mathrm{~nm}$ pulse is for pumping. These three laser pulses are introduced to the sample with incidence angle of about $45^{\circ}$ from the sample surface.

\section{Results and discussion}

Figure 2a shows the reflectivity spectra obtained below and above the transition temperature. By elevating the temperature, just the amplitude decreases almost uniformly. To assign the vibrational modes, the absorption spectrum is derived using the Lorentz model over a wider horizontal axis from 650 to $2500 \mathrm{~cm}^{-1}$. The obtained absorption peaks at 682, 942, 1069, 1281, 1551, and $2134 \mathrm{~cm}^{-1}$ originate from $a_{\mathrm{g}}$ vibrational modes of TCNQ, which are $\nu_{2}, \nu_{3}, \nu_{4}, \nu_{5}, \nu_{6}$, and $\nu_{7}$, respectively [1, 10-13]. These are induced by the dimerization [14]. A part of absorption spectrum is shown in Fig. 2c with a gray curve.

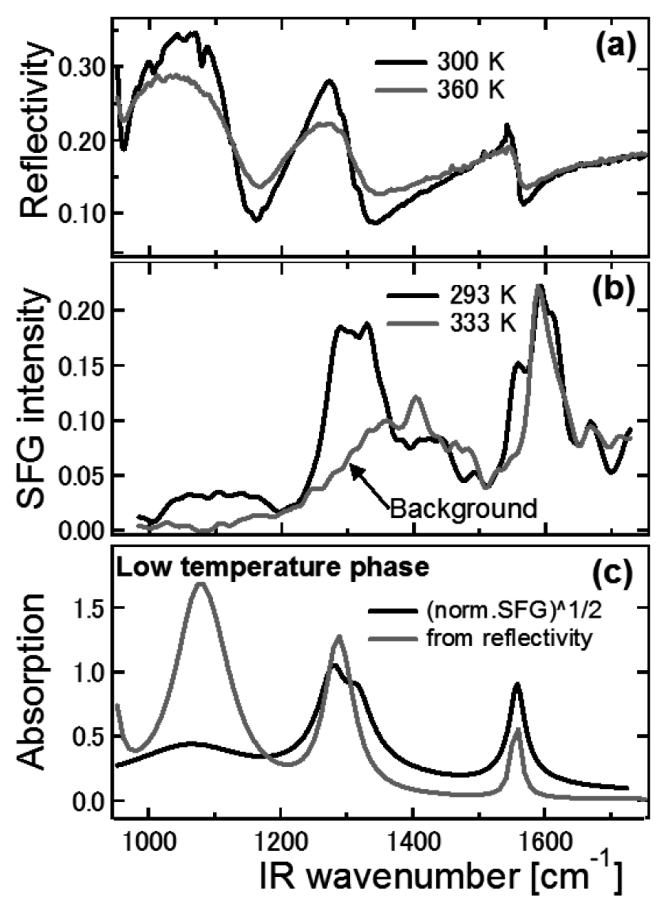

Fig. 2. (a) Linear reflectivity and (b) VSFG spectra obtained in the low temperature insulating and in the high temperature metal phases. (c) Derived absorption spectra in the low temperature phase.

VSFG spectrum at room temperature (a black curve in Fig. 2b) shows the vibrational modes at around $1100 \mathrm{~cm}^{-1}, 1300 \mathrm{~cm}^{-1}$, and $1550 \mathrm{~cm}^{-1}$, which are almost the same wave number as the reflectivity measurements. They almost disappear above the transition temperature (a gray curve), indicating that the TCNQ dimerization also exists at the surface and melts by the temperature, similar to bulk. A peak shown at $1600 \mathrm{~cm}^{-1}$ does not disappear by the temperature, which is due to the larger IR intensity and exists as the background.

VSFG spectra can be represented by $[15,16]$ :

$$
I\left(\omega_{\mathrm{SFG}}\right) \propto\left|\chi^{(2)}\right|^{2},
$$

where $\chi^{(2)}$ is composed of a non-resonance and a resonance parts as follows:

$$
\begin{aligned}
\chi^{(2)} & =\chi_{\mathrm{NR}}^{(2)}+\chi_{\mathrm{R}}^{(2)}\left(\omega_{\mathrm{IR}}\right) \\
= & \chi_{\mathrm{NR}}^{(2)}+\sum_{q} \frac{A_{q}}{\omega_{q}-\omega_{\mathrm{IR}}-\mathrm{i} \Gamma_{q}} .
\end{aligned}
$$

$A_{q}, \Gamma_{q}$, and $\omega_{q}$ are the amplitude, the width, and the wave number of modes, respectively. The resonant spectrum is deduced by subtracting the gray curve in Fig. $2 \mathrm{~b}$ from the black curve. The spectrum is fitted by Eq. (1) and shown in Fig. 2c with a black curve. We assumed that the vibrational modes almost disappeared at $333 \mathrm{~K}$, therefore we treated it as the non-resonance part $\left(\chi_{\mathrm{NR}}^{(2)}\right)$ in Eq. (2). Finally, the fitting is square-rooted in order to compare with the linear spectrum obtained by the re- 
flectivity. Similar absorption spectra were obtained from the surface and the bulk with peaks at the same wave number and the width, except for a peak at $1300 \mathrm{~cm}^{-1}$ which is composed with two peaks resolved only by VSFG (Fig. 2c). Absorption intensities in these spectra are different from each other, which may indicate the characteristic of phase transition occurring at the surface.

We have successfully performed the pump-probe VSFG experiments. Pump fluence is about three times larger than the other $800 \mathrm{~nm}$ pulse used to generate SFG signal. VSFG spectra before and after the pumping are shown in Fig. 3b. Similarly to the temperature dependent experiments, the absorption occurs at the same wave numbers and the reduction of the amplitude is also observed. The double peaks at around $1300 \mathrm{~cm}^{-1}$ are clearly shown and no change of the peak height at $1600 \mathrm{~cm}^{-1}$ is also reproduced. Dynamics of structural changes (reduction of the dimerization) is observed by delaying the pump pulse, when the wave number of IR pulse is fixed to $1310 \mathrm{~cm}^{-1}$ (Fig. 4).

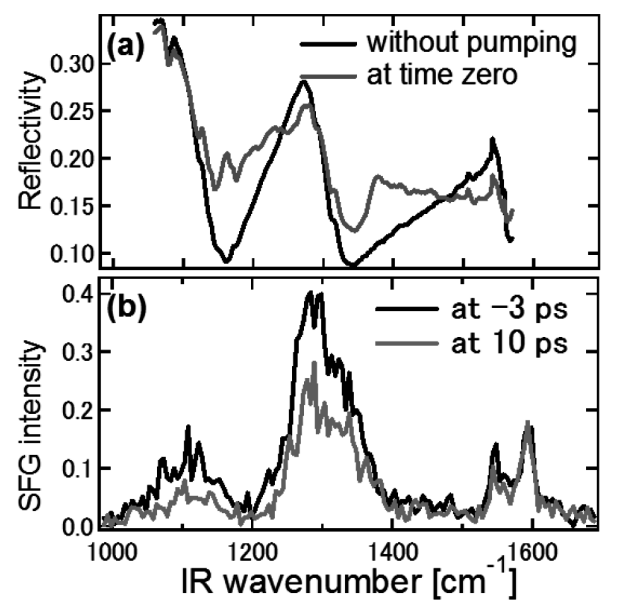

Fig. 3. Time-resolved reflectivity (a) and VSFG spectra (b) before and after the pumping.

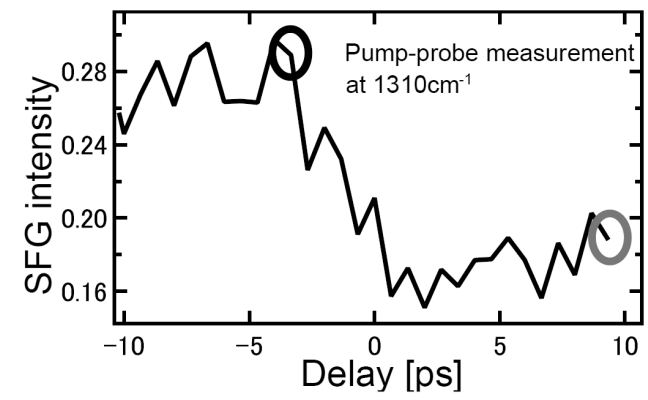

Fig. 4. Pump-probe VSFG experiments indicating the time-dependent degree of dimerization. The wave number of IR laser is fixed to $1310 \mathrm{~cm}^{-1}$.

On the contrary, for the reflectivity experiments in the pump-probe experiments, spectrum is shown in a differ- ent shape (a gray curve in Fig. 3a) which is not just a reduction of the amplitudes. One of the reasons is due to an experimental restriction to measure spectra in a wider wavenumber region, or it can be due to the different phase dynamics occuring at the surface and in the bulk.

\section{Conclusion}

We developed the VSFG equipment to investigate the structural dynamics at a solid surface and observed the photo-induced phase transition at the surface of an organic single crystal, $\beta^{\prime}$-(BEDF-TTF)(TCNQ). Dynamical change of degree of the TCNQ dimerization was observed in a vibrational spectroscopic view. These results were compared with the bulk properties obtained by reflectivity measurements and they shown basically a similar result. However, the spectral shape of reflectivity measurements was deformed and of less accuracy than the time-resolved VSFG spectra. These results may indicate the different dynamics occurring at the surface and in the bulk.

\section{References}

[1] Y. Iwasa, K. Mizuhashi, T. Koda, Y. Tokura, G. Saito, Phys. Rev. B 49, 3580 (1994).

[2] Y. Kawasugi, H.M. Yamamoto, N. Tajima, T. Fukunaga, K. Tsukagoshi, R. Kato, Phys. Rev. Lett. 103, 116801 (2009).

[3] R. Kumai, Y. Tokura, Chem. Lett. 28, 765 (1999).

[4] K. Yonemitsu, K. Nasu, Phys. Rep. 465, 1 (2008).

[5] T. Mori, H. Inokuchi, Solid State Commun. 59, 355 (1986).

[6] Y.R. Shen, The Principles of Nonlinear Optics, Wiley, New York 1984.

[7] K. Onda, M. Nakagawa, T. Asakai, R. Watase, A. Wada, K. Ichimaru, C. Hirose, J. Phys. Chem. 106, 3855 (2002).

[8] Y. Matsubara, T. Yoshida, T. Ishikawa, Y. Okimoto, S. Koshihara, K. Onda, Acta Phys. Pol. A 121, 340 (2012).

[9] H.M. Yamamoto, M. Hagiwara, R. Kato, Synth. Met. 133, 449 (2003).

[10] M. Uruichi, K. Yakushi, H.M. Yamamoto, R. Kato, J. Phys. Soc. Japan 75, 074720 (2006).

[11] R. Bozio, A. Girlando, C. Pecile, J. Chem. Soc., Faraday Trans. 2, 71, 1237 (1975).

[12] T. Mori, H. Inokuchi, Chem. Soc. Japan 60, 402 (1987).

[13] A. Girlando, C. Pecile, Spectrochim. Acta 29A, 1859 (1973).

[14] Y. Eto, A. Kawamoto, N. Matsunaga, K. Nomura, Phys. Rev. B 80, 174506 (2009).

[15] M. Buck, M. Himmelhaus, J. Vac. Sci. Technol. A 19, 2717 (2001).

[16] H. Noguchi, T. Okada, K. Onda, S.S. Kano, A. Wada, K. Domen, Surf. Sci. 528, 183 (2003). 